\title{
Intervenções de Enfermagem para idosos que envelheceram em instituições psiquiátricas: mapeamento cruzado
}

Tatiana Gomes da Silva ${ }^{1}$, Rosimere Ferreira Santana ${ }^{2}$, Priscilla Alfradique de Souza ${ }^{3}$

\author{
${ }^{1}$ Enfermeira. Discente do Programa de \\ Pós-Graduação em Enfermagem, nível \\ Mestrado, da Universidade Federal \\ Fluminense. Coordenadora de \\ enfermagem no Núcleo Assistencial Franco \\ da Rocha. Rio de Janeiro, RJ, Brasil. E-mail: \\ tatii-gomes@bol.com.br.

\footnotetext{
${ }^{2}$ Enfermeira, Doutora em Enfermagem. Professor Adjunto da Universidade Federal Fluminense. Niterói, RJ, Brasil. E-mail: rosifesa@enf.uff.br.

${ }^{3}$ Enfermeira, Doutora em Enfermagem. Professor Assistente da Universidade do Estado do Rio de Janeiro. Rio de Janeiro, RJ, Brasil. E-mail: priscillalfradique@gmail.com.
}

Recebido: 14/12/2015.

Aceito: 20/07/2016.

Publicado: 30/11/2016.

Como citar esse artigo:

Silva TG, Santana RF, Souza PA. Intervenções de Enfermagem para idosos que envelheceram em instituições psiquiátricas: mapeamento cruzado. Rev. Eletr. Enf. [Internet]. 2016 [acesso em: ____;18:e1185. Disponível em: http://dx.doi.org/10.5216/ree.v18.39049.

\begin{abstract}
RESUMO
Objetivou-se realizar o mapeamento cruzado de termos da linguagem de Enfermagem com o sistema de Classificação das Intervenções de Enfermagem, em prontuários de idosos com transtornos psiquiátricos. Trata-se de pesquisa documental, de mapeamento cruzado, com amostra intencional, composta por 30 prontuários de idosas com transtornos psiquiátricos. A coleta e a análise ocorreram entre julho de 2013 a maio de 2014, em três etapas. Foram encontradas 392 intervenções normalizadas, que resultaram em 33 intervenções, média de 13 por usuários, representadas por 15 intervenções do domínio comportamental e 10 do fisiológico básico. "Orientação para a realidade", "Melhora da socialização", " Assistência no autocuidado: atividades essenciais da vida diária" e "Apoio familiar" estiveram em 100\% dos prontuários. Foram descritas 16 atividades por intervenção. O mapeamento cruzado permitiu construir termos correspondentes às Intervenções de Enfermagem cotidianas em saúde mental, contribuindo para a prática assistencial.
\end{abstract}

Descritores: Saúde do Idoso; Cuidados de Enfermagem; Enfermagem Geriátrica; Enfermagem Psiquiátrica; Processos de Enfermagem.

\section{INTRODUÇÃO}

Pessoas com transtornos mentais crônicos envelhecem pelos mesmos motivos que o restante do substrato da população, ou seja, avanço tecnológico e científico ${ }^{(1)}$. Porém com uma relação diferenciada, já que alguns permaneceram grande parte de suas vidas reclusos em instituições psiquiátricas e estão habituados às rotinas, às normas, ao tratamento tradicional da doença psiquiátrica e aos seus sintomas ${ }^{(2-3)}$.

Atualmente, a implantação de um cuidado reabilitador, o programa de desinstitucionalização, 
principal eixo do trabalho desenvolvido em saúde mental, depara-se com a problemática da reinserção psicossocial de pessoas envelhecidas na instituição, com graves perdas da identidade pessoal e social ${ }^{(4)}$.

A progressiva transferência de pacientes para fora das instalações hospitalares requer indivíduos com autonomia e independência, para que eles recebam alta e passem a morar nas residências terapêuticas, e consigam conviver com outros usuários de serviços de saúde mental, recebendo ajuda financeira do poder público ${ }^{(5)}$. Similarmente, em gerontologia, o princípio fundamental do cuidado de enfermagem é a promoção da capacidade funcional, da autonomia e da independência - pilares de sustentação das ações de enfermagem ao idoso.

Consequentemente, o cuidado de enfermagem às pessoas que envelheceram nas instituições psiquiátricas é complexo, pois une-se às ações de conhecimento em gerontologia e em saúde mental. A assistência aos usuários que residem nos núcleos assistenciais volta-se para a ampliação de sua autonomia e de sua capacidade para o autocuidado; ao estímulo de suas funções cognitivas; e ao resgate de sua condição de cidadãos. Para tanto, essas instituições desenvolvem diferentes atividades terapêuticas e sociais, como oficinas, passeios de inserção social, visita a museus e viagens.

Todavia, o registro dessas ações nos prontuários tende a ser inferior ao praticado, pela complexidade assistencial. Há ainda a problemática de poucos estudos que detalham a linguagem padronizada de enfermagem na área de saúde mental, o que torna interessantes estudos que demonstrem sua aplicabilidade.

Dentre os diversos sistemas de classificação existentes para serem utilizados nas etapas do Processo de Enfermagem, destaca-se a Classificação das Intervenções de Enfermagem (NIC), que se refere às intervenções, alvo desse estudo. De abrangência mundial, essa taxonomia tem o intuito de documentar e comunicar o cuidado de enfermagem, por meio da integração de dados em sistemas informatizados, e proporcionar, na atualidade, uma fonte de dados para pesquisas ${ }^{(6-9)}$. De acordo com esse sistema de classificação, uma Intervenção de Enfermagem é definida como "qualquer tratamento que, baseado em julgamento e conhecimento clínico, um enfermeiro ponha em prática para intensificar os resultados do paciente" ${ }^{\prime(7)}$. Já as atividades de enfermagem são definidas como "condutas ou ações específicas tomadas para implementar uma intervenção e que auxiliam os pacientes a progredirem em direção a um resultado desejado"(7) ${ }^{(7)}$ As atividades de enfermagem situam-se no nível de concreto da ação, e uma série de atividades para implementar uma ação é necessária.

Logo, a relevância de estudos que tratam das Intervenções de Enfermagem no processo de desinstitucionalização dos usuários com transtornos psiquiátricos de longa duração, quando estruturadas em um sistema de classificação, deve permitir que pesquisas de enfermagem possam ser comparadas. Pode ainda melhorar a comunicação entre os enfermeiros e a equipe multiprofissional, com relação ao universo de atuação da enfermagem em saúde mental, e determinar a educação permanente dos profissionais dessa área, direcionada às ações que efetivamente são realizadas na prática e que contribuem para a melhoria do cuidado ao usuário. 
Diante do exposto, o presente estudo teve como objetivo realizar o mapeamento cruzado de termos da linguagem de Enfermagem com a NIC, em prontuários de idosos com transtornos psiquiátricos.

\section{MÉTODO}

Estudo de natureza aplicada, descritivo, de abordagem quantitativa, elaborado de acordo com o procedimento técnico da pesquisa documental, utilizando prontuários como fonte de coleta primária dos dados. Esse método foi escolhido por possibilitar a comparação linguística e semântica entre terminologias não padronizadas com o sistema de classificação eleito ${ }^{(6,8-10)}$.

O Instituto Municipal de Assistência a Saúde Juliano Moreira, na cidade do Rio de Janeiro/RJ, foi o cenário desta pesquisa. A equipe era multiprofissional e, dentre os cuidados com os usuários, incluia-se a orientação contextualizada e individualizada, preconizando-se a desinstitucionalização das pessoas.

Esse instituto contava com 11 enfermeiros e 50 técnicos de enfermagem, ambos divididos entre o serviço diurno e noturno. Cabia aos enfermeiros a prestação de cuidados integrais aos usuários, compondo, assim, a equipe multidisciplinar, constituída também por médicos clínicos e psiquiátricos, fisioterapeutas, psicólogos, nutricionistas, assistentes sociais e cuidadores informais. A principal função dessa equipe multidisciplinar era trabalhar a possibilidade de alta hospitalar por meio da articulação com as equipes do Centro de Atenção Psicossocial, dos serviços de Residências Terapêuticas e do Programa Federal de Volta pra Casa.

As dependências do Instituto Municipal de Assistência à Saúde Juliano Moreira eram constituídas por três núcleos assistenciais e uma unidade de pacientes agudos. A escolha do local levou em consideração o espaço físico de um dos núcleos assistenciais - especificamente dois lares de acolhimento desse núcleo, onde os usuários estão internados há mais de 40 anos.

A instituição passava por um processo de adequação do serviço de enfermagem, que tinha como premissa a implantação do Processo de Enfermagem. Dessa forma, o cuidado prestado ao paciente era registrado em um impresso próprio da unidade. Durante os três primeiros plantões de cada mês, os enfermeiros responsáveis por cada casa realizavam o exame físico e psíquico de cada paciente. A partir dos dados obtidos analisavam-se as reais necessidades das pacientes individualmente, realizando-se, então, os Diagnósticos e Intervenções de Enfermagem, que também eram registrados no impresso, denominado "plano de cuidados".

Cabiam aos enfermeiros a execução das Intervenções de Enfermagem, e a orientação e supervisão das atividades desenvolvidas pelos técnicos de enfermagem. Por isso, o registro das ações da equipe de enfermagem era abrangente.

Os enfermeiros registravam os cuidados prestados sem a preocupação do uso da linguagem padronizada. Para essa pesquisa, os autores consideraram os registros e o contexto que continham as Intervenções de Enfermagem descritas em linguagem livre. O período de coleta de dados foi desde o início da documentação em prontuário no impresso "plano de cuidados", datada de janeiro de 2011, até o início 
dessa pesquisa, em março de 2013.

Os participantes do estudo foram as 30 usuárias com transtornos psiquiátricos, que possuíam no mínimo seis evoluções nos planos de cuidados. Dessa forma, a população foi de 30 prontuários, os quais representavam $100 \%$ da população total. Destes, foram consideradas para este estudo as últimas seis evoluções de enfermagem, totalizando 392 evoluções de enfermagem analisadas.

Após a realização de teste piloto, para fins de treinamento e aprimoramento da pesquisadora na utilização do método, o mapeamento cruzado foi realizado no período de junho a dezembro de 2013 , em três etapas, a saber: (1) extração e normalização de termos; (2) separação e comparação dos termos não padronizados com os padronizados pela NIC; e (3) avaliação e refinamento do mapeamento ${ }^{(6,8-10)}$.

A primeira etapa foi realizada no período de julho a setembro de 2013. A pesquisadora realizou a extração eletrônica das informações que compuseram um banco de dados contendo os dados do paciente, o diagnóstico médico e o trecho das evoluções referentes ao contexto da intervenção exata. Por exemplo: no trecho da evolução no qual estava descrito "estimular atividades que valorizem a independência do paciente", os termos "independência do paciente" foram destacados após a fragmentação do trecho da evolução. Da mesma forma, no trecho no qual estava descrito: "orientado e auxiliado a usuária quanto à realização de higiene oral e corporal diariamente", os termos "orientado", "higiene oral" e "higiene corporal" foram colocados em destaque no banco de dados.

Os dados foram dispostos em uma planilha Excel para Windows. Após, foram normalizados, de acordo com a adequação de termos verbais, correção de ortografia, uniformização de sexo, número, exclusão das repetições, sinônimos e expressões casuais que não designam conceitos particulares.

No período de outubro de 2013 a janeiro de 2014, a pesquisadora procedeu à segunda etapa, por meio do mapeamento cruzado dos termos identificados na etapa anterior, com as Intervenções de Enfermagem da taxonomia NIC. Foram consideradas as seguintes regras do mapeamento cruzado: mapear o significado das palavras, não apenas as palavras; usar a palavra-chave na intervenção para mapear para a intervenção NIC; usar os verbos como as palavras-chaves na intervenção; mapear a intervenção partindo do rótulo da intervenção NIC para a atividade; manter a consistência entre a intervenção mapeada e a definição da intervenção NIC; usar o rótulo da intervenção NIC mais específico; e mapear as intervenções que tinham dois ou mais verbos para as duas ou mais intervenções NIC correspondentes ${ }^{(6)}$.

Os termos de enfermagem em linguagem livre foram comparados com as intervenções de NIC. Foi realizada a categorização dos termos de enfermagem, com análise de combinação, sendo que, quando o termo encontrado combinava exatamente com o termo do sistema de classificação, este era categorizado como combinação exata. Quando esse termo apresentava conceitos similares, sinônimos e termos relacionados, era categorizado como combinação parcial. Para apresentação dos resultados, as combinações exatas e parciais foram consideradas com o mesmo valor ${ }^{(6,8-10)}$.

Nos exemplos anteriormente citados, o termo "independência do paciente", extraído após a fragmentação do trecho da evolução considerado Intervenção de Enfermagem, foi considerado combinação 
exata com a NIC. Já os termos "orientada", "higiene oral" e "higiene corporal" foram considerados combinação parcial e correlacionados à intervenção padronizada da NIC "Assistência no Autocuidado: banho/higiene". Nesse caso, observa-se consistência entre o contexto da intervenção descrita no prontuário e a definição da intervenção proposta por NIC, que é: "Assistência a paciente para que faça a higiene pessoal”. No período de maio de 2014, a terceira etapa foi concretizada, avaliando-se e refinando o mapeamento cruzado. Nessa etapa, os dados levantados foram analisados por enfermeiros peritos, especialistas na área de Classificação de Enfermagem, de Psicogeriatria e Clínica Médico-Cirúrgica. Na seleção desses enfermeiros, consideraram-se a experiência prática mínima de cinco anos, o título de doutorado e a experiência em pesquisas sobre Sistemas de Classificação em Enfermagem. Por meio da concordância dos peritos, estabeleceu-se a relação entre os contextos das intervenções, os termos não padronizados e as intervenções de NIC. Essa etapa foi realizada em ciclos individual e em grupo. Já no segundo ciclo, houve o consenso dos peritos e, assim, não foi necessária a análise de concordância estatística.

Os dados foram analisados considerando sua frequência absoluta, percentual, média e desvio padrão. O desenvolvimento do estudo atendeu as normas nacionais e internacionais de ética em pesquisa envolvendo seres humanos (Parecer no 088/2013).

\section{RESULTADOS}

Por se tratar de uma instituição exclusivamente feminina, obteve-se $100 \%$ da população amostral de pacientes do sexo feminino, com idade média de 74,5 anos variando entre 60 a 89 anos. Conforme apresentado na Tabela 1, a amostra foi composta majoritariamente por idosas solteiras (87\%), analfabetas (93\%), naturais do Rio de Janeiro (40\%) e com tempo de internação entre 40 e 49 anos (53\%). Em relação à fonte de renda, a maioria das idosas (50\%) não possuía fundo de renda; para aquelas que o possuíam, o Benefício da Prestação Continuada da Lei Orgânica da Assistência Social (BPC/LOAS) foi o principal (27\%) evidenciado. 
Tabela 1: Característica sociodemográficas das idosas atendidas nas consultas de enfermagem, 30 prontuários. Rio de Janeiro, RJ, Brasil, 2014.

\begin{tabular}{|c|c|}
\hline Características & n (\%) \\
\hline \multicolumn{2}{|l|}{ Faixa etária, anos } \\
\hline $60-69$ & $6(20)$ \\
\hline $70-79$ & $17(57)$ \\
\hline $80-89$ & $7(23)$ \\
\hline \multicolumn{2}{|l|}{ Estado civil } \\
\hline Ignorado & $1(3)$ \\
\hline Casada & $1(3)$ \\
\hline Viúva & $2(7)$ \\
\hline Solteira & $26(87)$ \\
\hline \multicolumn{2}{|l|}{ Tempo de internação, anos } \\
\hline $10-20$ & $1(3)$ \\
\hline $30-39$ & $2(7)$ \\
\hline $40-49$ & $16(53)$ \\
\hline $50-59$ & $7(24)$ \\
\hline $60-69$ & $1(3)$ \\
\hline \multicolumn{2}{|l|}{ Naturalidade } \\
\hline Distrito Federal & $1(3)$ \\
\hline Sergipe & $2(7)$ \\
\hline Rio Grande do Norte & $2(7)$ \\
\hline São Paulo & $2(7)$ \\
\hline Minas Gerais & $7(21)$ \\
\hline Rio de Janeiro & $17(55)$ \\
\hline \multicolumn{2}{|l|}{ Fonte de renda } \\
\hline Pensão & $2(6)$ \\
\hline BAR & $5(17)$ \\
\hline BPC-LOAS & $8(27)$ \\
\hline Nada consta & $15(50)$ \\
\hline \multicolumn{2}{|l|}{ Grau de instrução } \\
\hline Ensino fundamental incompleto & $2(7)$ \\
\hline Analfabeto & $28(93)$ \\
\hline
\end{tabular}

BRA: Bolsa de Apoio a Ressocialização; BPC-LOAS: Benefício da Prestação Continuada da Lei Orgânica da Assistência Social.

Em se tratando das Intervenções de Enfermagem, dos 30 prontuários analisados, identificaram-se 392 intervenções normalizadas. Isso representou média de 13 intervenções por usuário. A maior concentração de intervenções era de orientação sobre as atividades de vida diária e para melhora na socialização. Após o cruzamento com a taxonomia e o refinamento, emergiram 33 intervenções equivalentes à terminologia NIC. Dessas, 11 (36\%) apresentaram frequência maior que 50\%. As intervenções "Assistência no autocuidado: atividades essenciais da vida diária", "Orientação para a realidade", "Melhora da socialização", "Apoio familiar" e "Manutenção do processo familiar", implementadas pelos enfermeiros em diferentes contextos, estiveram presentes em todos os prontuários e apresentaram média de repetição de 3,9 vezes. 
Tabela 2: Distribuição das Intervenções de Enfermagem equivalentes ao sistema de Classificação das Intervenções de Enfermagem (NIC), 30 prontuários. Rio de Janeiro, RJ, Brasil, 2014.

\begin{tabular}{|c|c|}
\hline Código/Intervenção NIC & n (\%) \\
\hline \multicolumn{2}{|l|}{ Domínio Comportamental } \\
\hline 4820 Orientação para a Realidade & $30(100)$ \\
\hline 5100 Melhora da Socialização & $30(100)$ \\
\hline 5440 Melhora do sistema de Apoio & $27(90)$ \\
\hline 4720 Estimulação Cognitiva & $27(90)$ \\
\hline 4920 Escutar Ativamente & $27(90)$ \\
\hline 5510 Educação em Saúde & $27(90)$ \\
\hline 4390 Terapia Socioambiental & $27(90)$ \\
\hline 4362 Modificação do Comportamento: habilidades sociais & $13(43)$ \\
\hline 4976 Melhora da Comunicacão: défice da fala & $11(36)$ \\
\hline 5220 Melhora da Imagem Corporal & $11(36)$ \\
\hline 5820 Redução da Ansiedade & $6(20)$ \\
\hline 5390 Melhora da Autopercepção & $6(20)$ \\
\hline 4978 Melhora da Comunicação: défice visual & $6(20)$ \\
\hline 4490 Assistência para parar de Fumar & $4(13)$ \\
\hline 5000 Construção de Relação Complexa & $4(13)$ \\
\hline \multicolumn{2}{|l|}{ Domínio Fisiológico Básico } \\
\hline 1805 Assistência no Autocuidado: atividades essenciais da vida diária & $30(100)$ \\
\hline 1710 Manutenção da Saúde Oral & $30(100)$ \\
\hline 1803 Assistência o Autocuidado: alimentação & $24(80)$ \\
\hline 1801 Assistência no Autocuidado: banho/higiene & $24(80)$ \\
\hline 1802 Assistência no Autocuidado: vestir/arrumar-se & $18(60)$ \\
\hline 0450 Controle de Constipação/Impactação & $13(43)$ \\
\hline 1850 Melhora do Sono & $12(40)$ \\
\hline 1860 Terapia de Deglutição & $10(40)$ \\
\hline 0610 Cuidados na Incontinência Urinária & $8(26)$ \\
\hline 0410 Cuidados na Incontinência Intestinal & $6(20)$ \\
\hline \multicolumn{2}{|l|}{ Domínio Fisiológico complexo } \\
\hline 3590 Supervisão da Pele & $17(56)$ \\
\hline 4040 Cuidados Cardíacos & $16(53)$ \\
\hline 4070 Precauções Circulatórias & $9(30)$ \\
\hline 2120 Controle da Hiperglicemia & $7(23)$ \\
\hline 3660 Cuidados com Lesões & $2(6)$ \\
\hline \multicolumn{2}{|l|}{ Domínio Família } \\
\hline 7140 Apoio Familiar & $30(100)$ \\
\hline 7130 Manutenção do Processo Familiar & $29(96)$ \\
\hline \multicolumn{2}{|l|}{ Domínio Segurança } \\
\hline 6490 Prevenção contra Quedas & $21(70)$ \\
\hline
\end{tabular}

Observaram-se, na padronização das intervenções, atividades tanto em saúde mental quanto em gerontologia. A temática de cada atividade esteve voltada para o estímulo à autonomia e à independência, para a construção do processo de desinstitucionalização. No Quadro 1 apresentam-se, de forma descritiva, as principais intervenções implementadas pelos enfermeiros com suas respectivas atividades, a fim de permitir melhor compreensão quanto ao papel do enfermeiro na saúde mental de idosos. Evidencia-se uma variedade de atividades (117 ao total), representando seis atividades implementadas por intervenção prescrita. 
Quadro 1: Atividades implementadas de acordo com a Intervenção de Enfermagem equivalente à terminologia Classificação das Intervenções de Enfermagem, analisadas pelas áreas Gerontologia e Saúde Mental, 30 prontuários.

\begin{tabular}{|c|c|c|}
\hline \multicolumn{3}{|c|}{ Intervenções em Saúde Mental } \\
\hline \multicolumn{2}{|l|}{ Intervenção/Definição } & Atividades \\
\hline \multicolumn{2}{|c|}{$\begin{array}{l}4820 \text { Orientação para a Realidade } \\
\text { Definição: promoção da percepção que tem o paciente } \\
\text { da identidade pessoal, do tempo e do ambiente }\end{array}$} & $\begin{array}{c}\text { Tratar o paciente pelo nome ao iniciar a interação } \\
\text { Informar o paciente sobre pessoa, local e tempo, se necessário } \\
\text { Apresentar a realidade de modo a preservar a dignidade do paciente } \\
\text { Incentivar ao paciente que use as próprias roupas; auxiliar quando } \\
\text { necessário }\end{array}$ \\
\hline \multicolumn{2}{|c|}{$\begin{array}{c}5100 \text { Melhora da Socialização } \\
\text { Definição: facilitação da capacidade de outra pessoa } \\
\text { para interagir com os outros }\end{array}$} & $\begin{array}{c}\text { Encorajar atividades sociais e comunitárias } \\
\text { Encorajar o respeito aos direitos dos outros } \\
\text { Encorajar melhoria do desenvolvimento em relações já criadas }\end{array}$ \\
\hline \multicolumn{2}{|c|}{$\begin{array}{l}4390 \text { Terapia Socioambiental } \\
\text { Definição: uso de pessoas, recursos e eventos do } \\
\text { ambiente do paciente para promover um adequado } \\
\text { funcionamento psicossocial }\end{array}$} & $\begin{array}{l}\text { Incluir o paciente nas decisões sobre seu próprio cuidado } \\
\text { Oferecer cuidados individualizados de enfermagem, conforme } \\
\text { apropriado } \\
\text { Encorajar o uso dos pertences pessoais } \\
\text { Oferecer livros, revistas e materiais artísticos e artesanais, conforme } \\
\text { as necessidades educativas, culturais e educacionais do paciente }\end{array}$ \\
\hline \multicolumn{2}{|c|}{$\begin{array}{l}5000 \text { Construção de Relação Complexa } \\
\text { Definição: estabelecimento de uma relação } \\
\text { terapêutica com o paciente para promover insight e } \\
\text { mudança de comportamento }\end{array}$} & $\begin{array}{l}\text { Estreitar a distância física entre o enfermeiro e o paciente, conforme } \\
\text { apropriado. } \\
\text { Estabelecer limites terapêuticos } \\
\text { Ajudar o usuário a identificar as áreas que necessitam ser abordadas } \\
\text { durante os grupos }\end{array}$ \\
\hline \multicolumn{3}{|c|}{ Intervenções em Gerontologia } \\
\hline \multicolumn{3}{|c|}{$\begin{array}{l}\text { Determinar a necessidade de assistência pelo indivíduo com as Atividades } \\
\text { Instrumentais da Vida Diária } \\
\text { Orientar o indivíduo sobre métodos alternativos de transporte } \\
\text { Providenciar técnicas para melhorar a cognição } \\
\text { Auxílio a pessoa para que estabeleça métodos e rotinas para cozinhar, limpar e } \\
\text { fazer compras } \\
\text { Orientar a pessoa sobre a armazenagem correta e segura dos alimentos } \\
\text { Encaminhar a serviços comunitários, se necessário }\end{array}$} \\
\hline $\begin{array}{l}6490 \text { Prevenção contra Quedas } \\
\text { Definição: instituição de precauções } \\
\text { especiais para paciente com risco de lesão } \\
\text { em decorrência de quedas }\end{array}$ & $\begin{array}{r}\text { Id } \\
\text { Identific } \\
\mathrm{Pa} \\
\mathrm{Enc} \\
\text { Orienta }\end{array}$ & $\begin{array}{l}\text { entificar comportamentos e fatores que afetem o risco de quedas } \\
\text { Ir características ambientais capazes de aumentar o potencial de quedas } \\
\text { tilhar com o paciente as observações sobre o modo de andar e de } \\
\text { movimentar-se } \\
\text { Providenciar dispositivos auxiliares (bengala e andador) } \\
\text { rajar o paciente a usar a bengala ou andador, conforme apropriado } \\
\text { r o paciente a chamar ajuda para movimentar-se, conforme apropriado } \\
\text { Providenciar iluminação adequada para aumentar a visibilidade }\end{array}$ \\
\hline $\begin{array}{l}1710 \text { Manutenção da Saúde Oral } \\
\text { Definição: manutenção e promoção de } \\
\text { higiene oral e saúde dentária a paciente } \\
\text { com risco de desenvolver lesões orais ou } \\
\text { dentárias }\end{array}$ & Orient & $\begin{array}{l}\text { Encorajar e auxiliar o paciente a enxaguar a boca } \\
\text { r e auxiliar o paciente a fazer a higiene oral após as refeições e sempre } \\
\text { que necessário } \\
\text { Auxiliar no cuidado de dentadura, quando necessário }\end{array}$ \\
\hline
\end{tabular}

Quanto às atividades que não foram passíveis de mapeamento, foram identificadas: "Atentar para a presença de equimoses e sangramentos (nasal ou de gengivas, fezes escuras, hematomas e hipotensão arterial)" e "Observar e registrar alteração psicomotora". A primeira atividade apresentava um termo abrangente, o que dificultou o mapeamento pela NIC. Já a segunda atividade esteve relacionada às alterações das atividades motoras causadas pelo transtorno psicótico, o que não houve correspondente na NIC. 


\section{DISCUSSÃO}

O principal dado desse estudo é a descrição padronizada das ações de enfermagem em saúde mental para pessoas institucionalizadas a longo prazo, pois retrata uma realidade complexa de cuidado, pautada em habilidades e conhecimento de intervenções dos domínios comportamental, fisiológico e familiar. Essas intervenções são utilizadas em conjunto pelos enfermeiros como principal ferramenta para o processo de desinstitucionalização. Uma proposta alternativa no cuidar em saúde mental que vá além da medicalização significa tratar o usuário em sua existência e em relação as suas condições concretas de vida ${ }^{(11-15)}$.

Com isso, cabe ressaltar que, dos sete domínios da NIC, somente dois não foram mapeados: "Sistemas de saúde" e "Comunidade"(7). Considera-se, contudo, que possam ter existido ações de enfermagem desenvolvidas nesses domínios, mas que não foram descritas no prontuário - talvez pela tradição de registro das ações individuais no prontuário.

No Domínio Comportamental, as Intervenções de Enfermagem relacionadas às questões sociais foram representativamente descritas. Desse modo, elas convergem com a premissa do enfermeiro de saúde mental que se permite estabelecer uma relação de confiança com o usuário, como descrito na intervenção "Construção de relação complexa". Nessa relação, prima-se pela autonomia do usuário, a construção de vínculos, pela construção dos limites terapêuticos e pelas atividades em grupo"(14).

Nesse contexto, os enfermeiros atuam como profissionais da equipe multidisciplinar e proporcionam atividades de reestruturação e inclusão na dinâmica global. Porém, não foram identificadas, no prontuário, intervenções que direcionassem o cruzamento com a NIC, como, por exemplo, o gerenciamento de caso.

No entanto, também predominou a relevância das Intervenções de Enfermagem relacionadas às famílias, identificadas pela ausência de apoio familiar, presente em $100 \%$ dos usuários, seguidas do rompimento de processos familiares, presente em $96,7 \%$. Neste segundo grupo de intervenções, somente uma idosa recebia visita dos familiares.

Os dados revelam a herança deixada por anos de uma assistência hospitalocêntrica, marcada pela segregação e pela desfiliação social de um contingente de usuários psiquiátricos que hoje são idosos ${ }^{(15-16)}$ e que perderam o convívio familiar há mais de 40 anos, o que se torna uma barreira na construção/reconstrução dos laços afetivos.

Assim, é necessário que o enfermeiro estabeleça uma relação de confiança com o usuário/família, orientando sobre a doença, o tratamento, o uso das medicações, as atividades terapêuticas, as demandas individuais e o direito ao atendimento na rede de serviço disponível na comunidade.

$\mathrm{Na}$ análise das atividades desenvolvidas com a família, evidenciam-se a realização de atividades de educação como fundamentais, e a compreensão contextual do usuário/família sobre a importância da promoção das atividades de vida diária com autonomia e independência. Orientar sobre os fatores relacionados tanto às alterações do processo psíquico quanto ao envelhecimento corroboram achados da complexidade do cuidar dessas pessoas ${ }^{(12,17-18)}$.

Outro dado de interesse se refere à maioria feminina e à idade - algumas eram muito idosas, com até 
89 anos. Duas hipóteses podem ser ponderadas: da maior rejeição das mulheres com transtornos mentais, tanto por serem as cuidadoras da família, ou pelo fato dos homens com transtornos mentais terem maior risco de mortalidade. Estudos descrevem que a longevidade no transtorno mental reside na resiliência, ou seja, na capacidade de resposta aos estressores causados por doenças e no modo como isso interfere na expectativa de vida ${ }^{(12,18)}$. Dessa forma, pelo seu progressivo aumento populacional, observa-se a necessidade de enfoque em estudos que abordem a saúde mental em idosos ${ }^{(18)}$.

Pesquisadores mostram que a ausência de dentição, a falta de hábitos em realizar a higiene oral, o processo de envelhecimento e a institucionalização são fatores que interferem diretamente no risco em desenvolver lesões orais ou dentárias. Logo, faz-se necessário que o enfermeiro promova intervenções voltadas para a manutenção da saúde oral dos usuários, orientando e auxiliando nas atividades relacionadas diretamente à saúde oral individualizada ${ }^{(12,18)}$.

Quanto às ocorrências de quedas, estudos comprovam que os idosos são os mais afetados, devido à perda de equilíbrio postural. As quedas podem ser decorrentes da diminuição das capacidades cognitivas, físicas e funcionais do idoso ${ }^{(19-20)}$. Nesse cenário, os enfermeiros atuam na identificação dos riscos para a queda e propõem, em reunião de equipe, o uso de dispositivos auxiliares, como bengala ou andador, se houver necessidade e tiver sido acordado com o usuário.

As atividades que não foram passíveis de mapeamento foram "Atentar para a presença de equimoses e sangramentos (nasal ou de gengivas, fezes escuras, hematomas e hipotensão arterial)" e "Observar e registrar alteração psicomotora". Na primeira atividade, os termos eram abrangentes, o que dificultou o mapeamento pela NIC, e a segunda atividade está relacionada às alterações das atividades motoras causadas pelo transtorno psicótico.

Pondera-se que, durante a análise dos registros, houve o reconhecimento de registros dos enfermeiros a respeito do seu papel no processo de desinstitucionalização e sua adequação à terminologia NIC, o que pode ter contribuído para sistematizar a assistência de enfermagem no âmbito da saúde mental.

Entretanto, vale destacar que ainda existe resistência dos enfermeiros quanto a utilização das taxonomias, por se tratar de termos contrários a proposta atual de trabalho na saúde mental, o que está de acordo com os dados encontrados nesse estudo. Desse modo, foram realizadas adequações dos termos da NIC como, por exemplo, alterando o termo "paciente" para "usuário", cujo foco está na prevenção da doença e na promoção da saúde, possibilitando o caminho em direção à comunidade, com qualidade e projetos de $\operatorname{vida}^{(21-22)}$.

Outros termos também foram adequados, como "monitorar e controlar" para "auxiliar e orientar", proporcionando, assim, direito à singularidade e ao respeito à especificidade de cada usuário. Entende-se que a assistência de enfermagem na saúde mental está além da técnica. Portanto, pode ser acordada e construída juntamente do usuário, e não imposta a ele. O termo "determinar" foi substituído por "discutir", já que as avaliações e as condutas adotadas para cada usuário são discutidas em reunião de equipe, o que favorece a troca multidisciplinar e a melhora na qualidade da assistência prestada ao usuário. 
Ainda sobre as adequações dos termos, a palavra "ajustar" foi alterada por "estreitar". A mudança nesse termo converge com pesquisas que mostram que o enfermeiro deve se permitir despir de algumas práticas e conceitos. Além disso, a enfermagem é entendida como uma relação humana entre uma pessoa que necessita de cuidados de serviço de saúde e um enfermeiro com uma formação especializada para reconhecer e responder às necessidades de ajuda ${ }^{(21,23)}$.

Sobre os termos não mapeados, vale discutir a ausência de correspondente para controle da agitação psicomotora, termo próprio da saúde mental e da psiquiatria. Recomenda-se detalhar suas atividades em uma Intervenção de Enfermagem para submissão e inclusão na taxonomia.

Apesar da limitação do estudo imposta pela escolha do mapeamento cruzado como ferramenta metodológica, por proporcionar dados coletados retrospectivamente e por profissionais de plantões diferentes, tanto do dia quanto da noite, a confiabilidade e a validade dos dados se deram pelas características peculiares do local estudado, com profissionais capacitados e especialistas em saúde mental, e também pelo registro sistemático no prontuário, que facilitou a coleta de dados. Reconhece-se que esse método é um instrumento potente para o processo de implantação da linguagem padronizada nos serviços de saúde, uma vez que permite aos enfermeiros comparar dados de forma consistente e generalizável ${ }^{(6-7,9,23)}$.

Contudo, evidenciou-se que os enfermeiros dos núcleos assistenciais cenários deste estudo demandam conhecimentos e habilidades no âmbito gerontológico e da saúde mental. Trata-se de um cuidar complexo, com amplitude em quase todos os domínios da Classificação das Intervenções de Enfermagem, embora haja limitação de discussão das intervenções com a literatura, por serem pouco descritas de forma objetiva e que permitam comparação com os achados deste estudo.

\section{CONCLUSÃO}

O estudo possibilitou identificar 33 Intervenções de Enfermagem no prontuário das idosas em uma instituição psiquiátrica de longo prazo, demonstrando a magnitude e a complexidade dos cuidados de enfermagem, além de habilidades comportamentais, como fisiológicas básicas e complexas.

Ao se compararem as intervenções descritas nos prontuários com as intervenções e atividades da Classificação das Intervenções de Enfermagem, reforça-se a necessidade de uma linguagem padronizada e de um instrumento, que direcione a assistência prestada, os quais podem facilitar a elaboração de futuras pesquisas comparativas da prática clínica de enfermagem na saúde mental.

As intervenções de enfermagem construídas para pessoas que envelheceram na instituição refletem a demanda do idoso no processo de reabilitação psicossocial e promoção do estímulo à realização de atividades de vida diária. Nesse sentindo, promover a socialização e o cuidado com a higiene oral e corporal são igualmente necessárias. Trata-se de idosas que sofreram perdas ao longo dos anos ocasionadas pela institucionalização, mas que merecem ser resgatadas, independentemente da idade biológica ou envelhecimento do humano. 


\section{REFERÊNCIAS}

1. Tramunt KG, Silva BTC, Nogueira LE, Ulrich EL, Bisol WL, Spanemberg L, et al. Perfil dos pacientes idosos internados na Unidade de Psiquiatria de um hospital universitário do sul do Brasil. Sci. Med. [Internet]. 2010 [acesso em: 6 jun 2016];20(4):289-91. Disponível em:

http://revistaseletronicas.pucrs.br/ojs/index.php/scientiamedica/article/viewFile/7661/5916.

2. Silva AL, Gomes AMT, Oliveira CD, Souza GG. Representações sociais do processo de envelhecimento de pacientes psiquiátricos institucionalizados. Esc. Anna Nery [Internet]. 2011 [acesso em: 613 jun 2016];15(1):124-31. Disponível em: http://www.redalyc.org/pdf/1277/127718940018.pdf.

3. Perlingeiro R. Os cuidados de saúde dos idosos entre as limitações orçamentárias e o direito a um mínimo existencial. R. Dir. Sanit. [Internet]. 2014 [acesso em: 6 jun 2016];15(1):83-118. Disponível em:

www.revistas.usp.br/rdisan/article/download/82808/85763.

4. Oliveira FB, Silva AO. Enfermagem em saúde mental no campo da reabilitação psicossocial e da interdisciplinaridade. Rev. Bras. Enferm. [Internet]. 2000 [acesso em: 6 jun 2016];53(4):584-92. Disponível em: http://www.scielo.br/scielo.php?script=sci_arttext\&pid=S0034-71672000000400013.

5. Lucena AF, Barros ALBL. Mapeamento cruzado: uma alternativa para a análise de dados em enfermagem. Acta Paul. Enferm. [Internet]. 2005 [acesso em: 6 jun 2016];18(1):82-8. Disponível em:

http://www.scielo.br/pdf/ape/v18n1/a11v18n1.pdf.

6. Mccloskey JC, Bulechek GM, Dochterman JM Classificação das Intervenções de enfermagem. 5a ed. Porto Alegre: Artmed; 2010.

7. Silva TN, Santana RF, Santos GLA, Silva LF, Giselle MB, Garcia TD. Intervenções de Enfermagem no programa de gerenciamento de crônicos: mapeamento cruzado. Rev. RENE. [Internet]. 2014 [acesso em: 6 jun 2016];15(6):9981006. Disponível em: http://www.revistarene.ufc.br/revista/index.php/revista/article/download/1838/pdf.

8. Sousa RM, Santo FHE, Santana RF, Lopes MVO. Diagnósticos de enfermagem identificados em pacientes oncohematólogicos: mapeamento cruzado. Esc Anna Nery. [Internet]. 2015 [acesso em: 6 jun 2016];19(1):54-65. Disponível em: http://www.scielo.br/scielo.php?script=sci_arttext\&pid=S1414-81452015000100054.

9. Cubas MR, Carvalho CMG, Malucelli A, Denipote AGM. Mapeamento dos termos do Eixo Ação entre diferentes classificações de enfermagem. Rev. Bras. Enferm [Internet]. 2011[acesso em: 13 jun 2016];64(2):248-53. Disponível em: http://www.scielo.br/pdf/reben/v64n2/a05v64n2.pdf.

10. Silva TG, Souza PA, Santana RF. Adequação da linguagem de enfermagem à prática com idosos residentes em uma instituição psiquiátrica de longa permanência: mapeamento cruzado. Rev. Pesqui. Cuid. Fundam. [Internet]. 2015 [acesso em: 6 jun 2016];7(4):3467-78. Disponível em: http://pesquisa.bvsalud.org/enfermagem/resource/pt/bde27204.

11. Faro ACM. Enfermagem em Reabilitação: ampliando os horizontes, legitimando o saber. Rev. Esc. Enferm. USP. [Internet]. 2006 [acesso em: 6 jun 2016];40(1):128-33. Disponível em:

http://www.scielo.br/pdf/reeusp/v40n1/a18v40n1.pdf.

12. Aquino MMB, Cavalcanti MT. Os dispositivos do lazer no contexto reforma psiquiátrica brasileira: o Clube de Lazer e Cidadania Colônia, um estudo de caso. Rev. Latinoam. Psicopat. Fund. [Internet]. 2004 [acesso em: 6 jun 2016];6(4):165-91. Disponível em:

http://www.psicopatologiafundamental.org/uploads/files/revistas/volume07/n4/os_dispositivos_de_lazer_no_conte xto_da_reforma_psiquiatrica_brasileira.pdf.

13. Campos CMS, Barros S. Reflexões sobre o processo de cuidar da enfermagem em saúde mental. Rev. Esc. Enferm. USP. [Internet]. 2000 [acesso em: 6 jun 2016];34(3):271-6. Disponível em:

http://www.scielo.br/scielo.php?script=sci_arttext\&pid=S0080-62342000000300008.

14. Fontes AP, Neri AL. Resilience in aging: literature review. Cien Saude Colet. 2015;20(5):1475-95.

15. Zauszniewski JA, Bekhet A, Haberlein S. A Decade of published evidence for psychiatric and mental health nursing interventions. Online J Issues Nurs. 2012;17(3):8.

16. Caldwell TM, Jorm AF. Mental health nurses' beliefs about interventions for schizophrenia and depression: a comparison with psychiatrists and the public. Aust N Z J Psychiatry. 2000;34(4):602-11.

17. Silva LA, Gomes AMT, Oliveira DC, Souza MGG. Representações sociais do processo de envelhecimento de pacientes psiquiátricos institucionalizados. Esc. Anna Nery Rev. Enferm. [Internet]. 2011 [acesso em: 6 jun 2016];15(1):124-31. Disponível em: http://www.scielo.br/scielo.php?script=sci_arttext\&pid=S1414- 


\section{8.}

18. Nasi C, Cardoso ASF, Schneider JF, Olschwsky A, Wetzel C. Conceito de integralidade na atenção em saúde mental no contexto da reforma psiquiátrica. Rev Min de Enferm [Internet]. 2009 [acesso em: 13 jun 2016];13(1). Disponível em: http://reme.org.br/artigo/detalhes/174.

19. Oliveira EMB, Lemes TA, Nóbrega JOT. Perfil dos idosos polimedicados internados na enfermaria da Clínica Médica do Hospital Regional de Samambaia, Distrito Federal. Acta de Ciências e Saúde. [Internet]. 2013 [acesso em: 6 jun 2016];2(1):8-20. Disponível em: www.Is.edu.br/actacs/index.php/ACTA/article/download/50/56.

20. Reinaldo AMS. Saúde mental na atenção básica como processo histórico de evolução da psiquiatria comunitária. Esc. Anna Nery Rev. Enferm. [Internet] 2008 [acesso em: 13 jun 2016];12(1):173-8. Disponível em: http://www.scielo.br/pdf/ean/v12n1/v12n1a27.pdf.

21. Soares RD, Villela JC, Borba LO, Brusamarello T, Maftum MA. O papel da equipe de enfermagem no centro de atenção psicossocial. Esc. Anna Nery [Internet]. 2011 [acesso em: 6 jun 2016];15(1):110-5. Disponível em: http://www.scielo.br/scielo.php?script=sci_arttext\&pid=S1414-81452011000100016.

22. Nóbrega MML, Garcia TR, Furtado LG, Albuquerque CC, Lima CDL. Terminologias de Enfermagem: da Taxonomia da NANDA à Classificação Internacional para a Prática de Enfermagem. Rev. Enferm. UFPE On Line [Internet]. 2008 [acesso em: 6 jun 2016];2(4),454-61. Disponível em:

http://www.revista.ufpe.br/revistaenfermagem/index.php/revista/article/viewFile/333/pdf 408.

23. Gonçalves AM, Sena RR. A reforma psiquiátrica no Brasil: contextualização e reflexos sobre o cuidado com o doente mental na família. Rev. Latino-am. Enfermagem. [Internet]. 2001 [acesso em: 6 jun 2016];9(2):48-55. Disponível em: http://www.revistas.usp.br/rlae/article/viewFile/1551/1596. 